Pacific Journal of Mathematics

PSEUDOCOMPACTNESS AND UNIFORM CONTINUITY IN 


\title{
PSEUDOCOMPACTNESS AND UNIFORM CONTINUITY IN TOPOLOGICAL GROUPS
}

\author{
W. W. Comfort and Kenneth A. Ross
}

This work contains a number of theorems about pseudocompact groups. Our first and most useful theorem allows us to decide whether or not a given (totally bounded) group is pseudocompact on the basis of how the group sits in its Weil completion. A corollary, which permits us to answer a question posed by Irving Glicksberg (Trans. Amer. Math. Soc. 90 (1959), 369-382) is: The product of any set of pseudocompact groups is pseudocompact. Following James Kister (Proc. Amer. Math. Soc. 13 (1962), 37-40) we say that a topological group $G$ has property $U$ provided that each continuous function mapping $G$ into the real line is uniformly continuous. We prove that each pseudocompact group has property $U$.

Sections 2 and 3 are devoted to solving the following two problems: (a) In order that a group have property $U$, is it sufficient that each bounded continuous real-valued function on it be uniformly continuous? (b) Must a nondiscrete group with property $U$ be pseudocompact? Theorem 2.8 answers (a) affirmatively. Question (b), the genesis of this paper, was posed by Kister (loc. cit.). For a large class of groups the question has an affirmative answer (see 3.1 ); but in 3.2 we offer an example (a Lindelöf space) showing that in general the answer is negative.

Much of the content of this paper is summarized by Theorem 4.1, in which we list a number of properties equivalent to pseudocompactness for topological groups. We conclude with an example of a metrizable, non totally bounded Abelian group on which each uniformly continuous real-valued function is bounded.

Conventions and definitions. All topological groups considered here are assumed to be Hausdorff. The algebraic structure of the groups we consider is virtually immaterial; in particular, our groups are permitted to be non-Abelian.

A topological group $G$ is said to be totally bounded if, for each neighborhood $U$ of the identity, a finite number of translates of $U$ covers $G$. It has been shown in [10] by Weil that each totally bounded group is a dense topological subgroup of a compact group and that this compactification is unique to within a topological isomorphism leaving $G$ fixed pointwise. We refer to this compactification of $G$ as the Weil 
completion of $G$, and we reserve the symbol $\bar{G}$ to denote it.

Kister's property $U$ was defined in the summary above. In the same vein, we say that a topological group has property $B U$ if each bounded continuous real-valued function on $G$ is uniformly continuous. The uniform structure on $G$ referred to implicitly in the definitions of properties $U$ and $B U$ should be taken to be either the left uniform structure, defined as in 4.11 of [7], or the right uniform structure. It often happens that these structures do not coincide, and in this case there is a left uniformly continuous real-valued function on $G$ which is not right uniformly continuous. Nevertheless it is easy to see that every [bounded] continuous real-valued function on $G$ is left uniformly continuous if and only if every [bounded] continuous real-valued function on $G$ is right uniformly continuous. Hence the definitions of properties $U$ and $B U$ are unambiguous.

Our topological vocabulary is that of the Gillman-Jerison text [5]. The following definition, which is useful in $\S 2$, is in consonance with $4 J$ of [5]: A topological space is a $P$-space provided that each of its $G_{\delta}$ subsets is open.

1. Pseudocompact groups. The Weil completion of a topological group plays a fundamental rôle in many of the arguments which follow. Our first result shows that each pseudocompact group admits such a completion.

THEOREM 1.1. Each pseudocompact group is totally bounded.

Proof. If the topological group $G$ is not totally bounded, then there is a neighborhood $U$ of the identity $e$ in $G$ and a sequence $\left\{x_{k}\right\}$ of points in $G$ for which

$$
x_{k} \notin \bigcup_{n<k} x_{n} U
$$

for all $k$. We choose a symmetric neighborhood $V$ of $e$ for which $V^{4} \subset U$, and we select for each positive integer $k$ a nonnegative continuous function $f_{k}$ on $G$ such that

$$
f_{k}\left(x_{k}\right)=k \text { and } f_{k} \equiv 0 \text { off } x_{k} V \text {. }
$$

Using the local finiteness of the sequence $\left\{x_{k} V\right\}$, it is easy to check that the real-valued function $f$ defined on $G$ by the relation

$$
f(x)=\sum_{k=1}^{\infty} f_{k}(x)
$$

is continuous. Since $f$ is unbounded, the group $G$ is not pseudocompact. 
Discussion. Although pseudocompact groups have not (so far as we can determine) been in their own right an object of detailed study, various authors have considered an example in one connection or another. If $\left\{X_{\alpha}\right\}_{\alpha \in A}$ is a set of separable metric spaces - which we here take to be topological groups - then the set

$$
\begin{array}{r}
Y=\left\{x \in \Pi X_{\alpha}: x_{\alpha} \text { is the identity in } X_{\alpha}\right. \text { for all } \\
\text { but countably many } \alpha \text { in } A\}
\end{array}
$$

is an example of what Corson in [3] calls a $\Sigma$-space. Corson shows in his Theorem 2 that each continuous real-valued function on $Y$ admits a continuous extension to $\Pi X_{\alpha}$. It follows that if each $X_{\alpha}$ is compact, then $Y$ is pseudocompact and $\Pi X_{\alpha}$ is the Stone-Čech compactification of $Y$. This and other interesting results were obtained (also in the product-space context) by Glicksberg in [6]. Kister examined in [8] the case in which each $X_{\alpha}$ is a compact topological group.

Like every pseudocompact space, the $\Sigma$-space $Y$ defined above meets each nonempty $G_{\delta}$ subset of its Stone-Čech compactification. The appropriate group-theoretic analogue of this topological characterization of pseudocompactness is given in the following theorem. The reader will notice instantly that this theorem yields information about the Stone-Cech compactification of a pseudocompact group; we shall incorporate this observation into Theorem 4.1.

The Baire sets in a topological space $X$ are those subsets of $X$ belonging to the smallest $\sigma$-algebra containing all zero-sets in $X$.

TheOREM 1.2. Let $G$ be a totally bounded group and let

$$
\begin{aligned}
\mathscr{N}= & \{N: N \text { is a closed, normal subgroup of } \bar{G} \text { and } \\
& \left.N \text { is a } G_{\delta} \text { set in } \bar{G}\right\} .
\end{aligned}
$$

Then the following assertions are equivalent:

(a) $G$ is pseudocompact;

(b) each translate of each element of $\mathscr{N}$ meets $G$;

(c) each nonempty Baire subset of $\bar{G}$ meets $G$;

(d) each nonempty $G_{\delta}$ subset of $\bar{G}$ meets $G$;

(e) each continuous real-valued function on $G$ admits a continuous extension to $\bar{G}$.

Proof. $\quad(a) \Rightarrow$ (b). If (b) fails, then $x_{0} N \cap G=\varnothing$ for some $x_{0}$ in $\bar{G}$ and some $N$ in $\mathscr{N}$. Since $N$ is clearly not open, the quotient group $\bar{G} / N$ is infinite. Like any compact, first countable group, $\bar{G} / N$ is metrizable. Choosing an unbounded real-valued continuous function $f$ on $\bar{G} / N \backslash\left\{x_{0} N\right\}$ and defining $g$ on $\bar{G} \backslash x_{0} N$ by the relation

$$
g(x)=f(x N) \text {, }
$$


we see that $g$ is unbounded and continuous. The restriction of $g$ to $G$ is unbounded, and hence (a) fails.

(b) $\Rightarrow(c)$. This implication follows trivially from the following fact, a special case of Lemma 2.4 of [9]: If $E$ is a Baire subset of $\bar{G}$, then $E=E N$ for some $N$ in $\mathscr{N}$.

$(c) \Rightarrow(d)$. Since $\bar{G}$ is completely regular, each nonempty $G_{\delta}$ subset of $\bar{G}$ contains a nonempty zero-set of $\bar{G}$.

$(d) \Rightarrow(b)$. This is clear.

(b) $\Rightarrow($ e). Let $f$ be a real-valued continuous function on $G$, and let $\mathscr{B}$ be a countable base for the topology on the line. For each $B$ in $\mathscr{B}$ there is clearly an open subset $U_{B}$ of $\bar{G}$ for which

$$
f^{-1}(B)=U_{B} \cap G \text {. }
$$

By 1.6 and 2.4 of [9], there is an element $N_{B}$ of $\mathscr{N}$ for which

$$
c l_{\bar{G}} U_{B}=N_{B} \cdot c l_{\bar{G}} U_{B} \text {. }
$$

Setting $N=\bigcap_{B \in \mathscr{A}} N_{B}$, we clearly have $N \in \mathcal{N}$ and $c l_{\bar{G}} U_{B}=N \cdot c l_{\bar{G}} U_{B}$ for each $B$ in $\mathscr{B}$.

We next prove:

$\left(^{*}\right)$ If $x_{1} \in G, x_{2} \in G$, and $x_{1}^{-1} x_{2} \in N$, then $f\left(x_{1}\right)=f\left(x_{2}\right)$.

If $\left(^{*}\right)$ fails, we can find neighborhoods $B_{1}$ and $B_{2}$ of $f\left(x_{1}\right)$ and $f\left(x_{2}\right)$ respectively such that $B_{1} \in \mathscr{B}, B_{2} \in \mathscr{B}$, and $c l B_{1} \cap c l B_{2}=\varnothing$. Since $f$ is continuous on $G$, we have

$$
c l_{G} f^{-1}\left(B_{1}\right) \cap c l_{G} f^{-1}\left(B_{2}\right)=\varnothing,
$$

i.e.,

$$
c l_{G}\left(U_{B_{1}} \cap G\right) \cap \operatorname{cl}_{G}\left(U_{B_{2}} \cap G\right)=\varnothing .
$$

Now $x_{1} \in N \cdot c l_{G}\left(U_{B_{1}} \cap G\right)$; hence

$$
x_{2} \in N \cdot c l_{G}\left(U_{B_{1}} \cap G\right) \subset N \cdot \operatorname{cl}_{\bar{G}}\left(U_{B_{1}}\right)=\operatorname{cl}_{\bar{G}}\left(U_{B_{1}}\right),
$$

so that $x_{2} \in c l_{G}\left(U_{B_{1}} \cap G\right)$. Of course $x_{2} \in \operatorname{cl}_{G i}\left(U_{B_{2}} \cap G\right)$, and this contradiction completes the proof of $(*)$.

With $\left(^{*}\right)$ and hypothesis (b) at our disposal, it is easy to define an extension $\bar{f}$ of $f$ : given $x_{0}$ in $\bar{G}$, we choose any $x$ in $x_{0} N \cap G$ and set $\bar{f}\left(x_{0}\right)=f(x)$.

To check the continuity of $\bar{f}$ at an arbitrary point $x_{0}$ in $\bar{G}$, we choose $\varepsilon>0$. We will produce a neighborhood $U$ of the identity in $\bar{G}$ with the property that $\left|\bar{f}\left(x_{0}\right)-\bar{f}\left(y_{0}\right)\right|<\varepsilon$ whenever $y_{0} \in x_{0} U$. Indeed, choose $x \in x_{0} N \cap G$ and let $V$ be a neighborhood of the identity in $\bar{G}$ such that

$$
|f(x)-f(y)|<\varepsilon \text { whenever } y \in x V \cap G \text {. }
$$


Now let $U$ be any neighborhood of the identity in $\bar{G}$ for which $U^{2} \subset V$. It is easy to see (directly, or by 8.7 of [7]) that there is an $M$ in $\mathcal{r}$ such that $M \subset U \cap N$. Now for any point $y_{0}$ in $x_{0} U$ there is (again by hypothesis (b)) a point $z$ in $\left(x_{0}^{-1} y_{0} M\right) \cap G$. Since $z \in x x_{0}^{-1} y_{0} N \subset N y_{0} N=$ $y_{0} N$, we have $\bar{f}\left(y_{0}\right)=f(z)$. And since $z \in x x_{0}^{-1} y_{0} M \subset x U M \subset x U^{2} \subset x V$, it follows that $\left|\bar{f}\left(x_{0}\right)-\bar{f}\left(y_{0}\right)\right|=|f(x)-f(z)|<\varepsilon$. Hence $U$ is as desired and $\bar{f}$ is continuous at $x_{0}$.

$(\mathrm{e})=$ (a). Since every continuous real-valued function with domain $\bar{G}$ is bounded, this implication is obvious.

1.3. Discussion. If $\left(G_{0}, \mathcal{T}\right)$ is a compact group and $\mathscr{N}$ denotes the family of subgroups of $G_{0}$ defined as in the hypothesis of Theorem 1.2 , then the collection of translates of elements of $\mathscr{N}$ clearly constitutes a base for a $P$-space topology $\mathscr{P}$ on $G_{0}$. Since any $G_{\delta}$ set in $G_{0}$ that contains the identity must contain a member of $\mathscr{N}, \mathscr{P}$ is the smallest $P$-space topology containing $\mathscr{T}$. In fact,

$\mathscr{P}=\left\{U: U\right.$ is a countable intersection of $\mathscr{T}$-open subsets of $\left.G_{0}\right\}$.

Using these observations and 1.2, we have the following fact: A (dense) subgroup $G$ of $G_{0}$ is pseudocompact if and only if $G$ is $\mathscr{P}$-dense in $G_{0}$.

Gillman and Jerison present in 9.15 of [5] an example (due to Novák-Terasaka) of a pseudocompact space $X$ for which $X \times X$ is not pseudocompact. In the positive direction, a number of authors (see especially [6] and [4]) have given various conditions on a family of pseudocompact spaces sufficient to ensure that the product be pseudocompact.

THEOREM 1.4. The product of any set of pseudocompact groups is pseudocompact.

Proof. Let the set $A$ index the family $\left\{G_{\alpha}\right\}_{\alpha \in A}$ of pseudocompact groups, and let

$$
G=\prod_{\alpha \in A} G_{\alpha}
$$

The uniqueness aspect of Weil's theorem assures us that the compact group $\prod_{\alpha \in A} \overline{G_{\alpha}}$ is (homeomorphic with) $\bar{G}$. According to 1.2 , then, we need only show that each nonempty $G_{\delta}$ subset of $\prod_{\alpha \in A} \overline{G_{\alpha}}$ hits $G$.

Let $U$ be such a set, say $U=\bigcap_{n=1}^{\infty} U_{n}$ where each $U_{n}$ is a basic set of the form

$$
U_{n}=\prod_{\alpha \in A} U_{n, \alpha} ;
$$

here each $U_{n, \alpha}$ is open in $\overline{G_{\alpha}}$, and for each $n$ we have $U_{n, \alpha}=\overline{G_{\alpha}}$ for 
all but finitely many $\alpha$ in $A$. Let

$$
V_{\alpha}=\bigcap_{n=1}^{\infty} U_{n, \alpha}
$$

Then $V_{\alpha}$ is a nonempty $G_{\delta}$ set in $\overline{G_{\alpha}}$, and thus by 1.2 there is a point $x_{\alpha}$ in $V_{\alpha} \cap G_{\alpha}$. Evidently the point of $\bar{G}$ whose $\alpha$ coordinate is $x_{\alpha}$ lies in $U \cap G$.

In what follows we will consider at length Kister's question "Must a nondiscrete group with property $U$ be pseudocompact?" We now quickly handle the converse question.

THEOREM 1.5. Every pseudocompact group has property $U$.

Proof. If $f$ is a continuous real-valued function on the pseudocompact group $G$, then by 1.1 and $(\mathrm{a}) \Rightarrow(\mathrm{e})$ of $1.2, f$ admits a continuous extension $\bar{f}$ on $\bar{G}$. Since $\bar{f}$ is uniformly continuous on $\bar{G}$, it follows that $f$ is uniformly continuous on $G$.

2. Property $B U$ implies property $U$. This theorem is proved in 2.8. Our key lemma is 2.2.

LEMMA 2.1. If the topological group $G$ is not a P-space, then some nonempty $G_{\delta}$ subset $H$ of $G$ has no interior. The set $H$ may be chosen to be a closed subgroup.

Proof. There is a sequence $\left\{V_{k}\right\}$ of neighborhoods of $e$ for which $e \in \operatorname{int} \bigcap_{k=1}^{\infty} V_{k}$. Selecting a sequence $\left\{U_{k}\right\}$ of symmetric neighborhoods of $e$ such that $U_{k+1}^{2} \subset U_{k} \cap V_{k}$ and defining $H=\bigcap_{k=1}^{\infty} U_{k}$, we see (directly, or from 5.6 of [7]) that the $G_{\delta}$ set $H$ is a closed subgroup of $G$. Being a subgroup that is not open, $H$ has no interior.

Theorem 2.2. If the topological group $G$ has property $B U$, then $G$ is totally bounded or $G$ is a P-space.

Proof. Suppose the conclusion fails. Since $G$ is not a $P$-space, there is a sequence $\left\{U_{k}\right\}$ of neighborhoods of $e$ for which int $\bigcap_{k=1}^{\infty} U_{k}=\varnothing$. Since $G$ is not totally bounded, there is, just as in the proof of 1.1, a neighborhood $V$ of $e$ and a sequence $\left\{x_{k}\right\}$ of points in $G$ such that the sequence $\left\{x_{k} V\right\}$ is locally finite and pairwise disjoint.

For each integer $k$ there is a continuous function $f_{k}$ on $G$ for which $f_{k}\left(x_{k}\right)=1, f_{k} \equiv 0$ off $x_{k}\left(V \cap U_{k}\right)$, and $0 \leqq f_{k} \leqq 1$. The function $f=\sum_{k=1}^{\infty} f_{k}$ is bounded and continuous on $G$, and hence is (left) uniformly continuous. Thus there is a neighborhood $W$ of $e$ for which. $|f(x)-f(y)|<1$ whenever $x^{-1} y \in W$. We may take $W \subset V$. Since 
int $W \neq \varnothing$, we cannot have $W \subset \bigcap_{k=1}^{\infty} U_{k}$. Thus there is an integer $m$ and a point $p$ for which $p \in W \backslash U_{m}$. Now $x_{m}^{-1}\left(x_{m} p\right) \in W$, so that $\left|1-f\left(x_{m} p\right)\right|=\left|f\left(x_{m}\right)-f\left(x_{m} p\right)\right|<1$ and we have $f\left(x_{m} p\right) \neq 0$. Thus $x_{m} p \in \bigcup_{k} x_{k}\left(V \cap U_{k}\right)$. Since $x_{m} V \cap x_{k} V=\varnothing$ whenever $k \neq m$, we must have $x_{m} p \in x_{m}\left(V \cap U_{m}\right)$. But then $p \in U_{m}$, a contradiction completing the proof.

Our next result, used in the proof of 2.4, is given here in considerable generality because of its application in connection with Example 3.2.

THEOREM 2.3. Let the topological group $G$ be a P-space. Then the following are equivalent:

(a) $G$ has property $U$;

(b) $G$ has property $B U$;

(c) the characteristic function of every open-and-closed subset of $G$ is uniformly continuous.

Proof. Only the implication (c) $\Rightarrow$ (a) requires proof. Given a continuous real-valued function $f$ on $G$, we note that for each rational pair $(a, b)$, with $a \leqq b$, the set $f^{-1}([a, b])$ is closed; being a $G_{\delta}$ set in $G$, this set is also open. Since the characteristic function $\psi_{f^{-1}([a b])}$ is left uniformly continuous, there is a neighborhood $U_{a, b}$ of $e$ such that $x^{-1} y \in U_{a, b}$ implies $\left|\psi_{f^{-1}\{[a b]}(x)-\psi_{\left.f^{-1}[a b]\right\}}(y)\right|<1$. That is, $x^{-1} y \in U_{a, b}$ implies that $x \in f^{-1}([a, b])$ if and only if $y \in f^{-1}([a, b])$. Let $U=$ $\cap\left\{U_{a, b}: a, b\right.$ rational and $\left.a \leqq b\right\}$; then $U$ is a neighborhood of $e$ since $G$ is a $P$-space. To establish the left uniform continuity of $f$ it will clearly suffice to show that $f(x)=f(y)$ whenever $x^{-1} y \in U$. Suppose then that $x^{-1} y \in U$ and that $f(x)=p$. For appropriate sequences $\left\{a_{k}\right\}$ and $\left\{b_{k}\right\}$ of rational numbers, we have $\{p\}=\bigcap_{k}\left[a_{k}, b_{k}\right]$. Then $x \in f^{-1}\left(\left[a_{k}, b_{k}\right]\right)$ for all $k$. Since $x^{-1} y \in U_{a_{k}, b_{k}}$ for all $k$, we have $y \in \bigcap_{k} f^{-1}\left(\left[a_{k}, b_{k}\right]\right)=$ $f^{-1}(\{p\})$ and $f(y)=p=f(x)$.

COROLlaRY 2.4. If the topological group $G$ has property $B U$ and is not totally bounded, then $G$ has property $U$.

Proof. By Theorem 2.2, $G$ is a $P$-space. The result now follows from 2.3.

Corollary 2.4 gives an affirmative answer to problem (a) of the introduction for groups which are not totally bounded. The trick which handles the totally bounded situation consists, roughly speaking, in reducing to the metrizable case (where the proof is easy).

Lemma 2.5. If a topological group $G$ is metrizable and has property $B U$, then $G$ is compact or discrete. 
Proof. This is immediate from Atsuji's Theorem 1 in [1]. For a direct proof (by contradiction), assume otherwise and note that by Theorem 2.2, $G$ must be totally bounded. Since $G$ is not compact, $G$ is not complete. Hence there is a nonconvergent Cauchy sequence $\left\{x_{k}\right\}$ in $G$. By Tietze's theorem the function mapping $x_{k}$ to $(-1)^{k}$ can be extended to a real-valued continuous function bounded on $G$, and this bounded function is obviously not uniformly continuous.

LEMMA 2.6. If $G$ is a topological group with property $B U$, and if $H$ is a closed normal subgroup of $G$, then $G / H$ has property $B U$.

Proof. Let $f$ be a bounded continuous real-valued function on $G / H$, and let $\varepsilon>0$. Denoting by $\pi$ the natural projection of $G$ onto $G / H$, we note that $f \circ \pi$ is left uniformly continuous on $G$. Hence there is a neighborhood $V$ of $e$ for which

$$
|f \circ \pi(x)-f \circ \pi(y)|<\varepsilon \text { whenever } x^{-1} y \in V .
$$

Of course $\pi(V)$ is a neighborhood of $H$ in $G / H$. Now suppose that $(x H)^{-1}(y H) \in \pi(V)$. Then $x^{-1} y H=v H$ for some $v \in V$, so that $x^{-1} y h=$ $v$ for some $h \in H$. Then $x^{-1}(y h) \in V$ and therefore

$$
\begin{aligned}
|f(x H)-f(y H)| & =|f \circ \pi(x)-f \circ \pi(y)| \\
& =|f \circ \pi(x)-f \circ \pi(y h)|<\varepsilon .
\end{aligned}
$$

That is, $f$ is left uniformly continuous.

THEOREM 2.7. Let $G$ be a totally bounded group with property $B U$. Then $G$ is pseudocompact.

Proof. If $G$ is not pseudocompact, then according to 1.2 there is a point $p$ in $\bar{G}$ and a closed normal subgroup $N$ of $\bar{G}$ such that $G \cap p N=\varnothing$ and $\bar{G} / N$ is metrizable. Since $p N \notin G N / N$ and $G N / N$ is the continuous image of $G N$ under the natural projection, $G N / N$ is a dense proper subgroup of $\bar{G} / N$. Since a discrete subgroup of a topological group is closed (see 5.10 of [7]), it follows that $G N / N$ is a nondiscrete, noncompact metrizable group.

It is clear that any group, one of whose dense subgroups has property $B U$, must itself have property $B U$. In particular the group $G N$; in which $G$ is dense, has property $B U$. Hence $G N / N$ has property $B U$ by 2.6 , and $G N / N$ does not have property $B U$ by 2.5. This contradiction completes the proof.

THEOREM 2.8. A topological group has property $B U$ if and only if it has property $U$. 


\section{Proof. Use 2.4 and 2.7.}

3. Kister's question. We first give a partial affirmative answer to the question posed by Kister in [8].

THEOREM 3.1. If the topological group $G$ has property $B U$ and is not a $P$-space, then $G$ is pseudocompact.

Proof. The group $G$ is totally bounded by 2.2 , and hence is pseudocompact by 2.7 .

EXAMPLE 3.2. We now give an example of a nondiscrete topological Abelian group that is a $P$-space and has property $U$. Such a group is clearly not pseudocompact: every pseudocompact $P$-space is finite. Hence this example shows that Kister's question mentioned in the summary has a negative answer.

Let $A$ be an index set of cardinality $\aleph_{1}$ and let $G$ consist of all elements $x$ in $\prod_{\alpha \in A}\{1,-1\}_{\alpha}$ such that $x_{\alpha}=1$ for all but finitely many coordinates $\alpha$. Let $\Omega$ be the first uncountable ordinal and well-order $A$ according to the order-type $\Omega: A=\{\alpha: \alpha<\Omega\}$. For $\alpha \in A$, let

$$
H_{\alpha}=\left\{x \in G: x_{\beta}=1 \text { for all } \beta<\alpha\right\} \text {. }
$$

We decree that the subgroups $H_{\alpha}$ and each of their translates be open and thereby obtain a basis for a topology under which $G$ is a topological group. Clearly $G$ is a $P$-space and $G$ is not discrete.

We shall show that $G$ has property $U$. By Theorem 2.3 we need show only that the characteristic function $\psi_{W}$ of an open-and-closed set $W$ is uniformly continuous. For $\alpha \in A$, let $W_{\alpha}=U\left\{x H_{\alpha}: x H_{\alpha} \subset W\right\}$. Evidently $\left\{W_{\alpha}\right\}_{\alpha<\Omega}$ is a nondecreasing family of open-and-closed sets, and $U_{\alpha<\Omega} W_{\alpha}=W$. Since $\psi_{W_{\alpha}}(x)=\dot{\psi}_{W_{\alpha}}(y)$ whenever $x^{-1} y \in H_{\alpha}$, the characteristic function of each $W_{\alpha}$ is uniformly continuous. Hence it suffices to show that $W=W_{\alpha}$ for some $\alpha$.

Assume that $W \neq W_{\alpha}$ for all $\alpha$, and let

$$
V_{\alpha}=\cup\left\{x H_{\alpha}: x H_{\alpha} \cap W \neq \varnothing \text { and } x H_{\alpha} \cap(G \backslash W) \neq \varnothing\right\} \text {. }
$$

It is easy to see that each $V_{\alpha}$ is nonvoid and that $V_{\alpha} \supset V_{\gamma}$ whenever $\alpha<\gamma<\Omega$. It suffices now to prove that $\cap_{\alpha<\Omega} V_{\alpha}$ is nonvoid, since any element in this intersection belongs to the closures of both $W$ and $G \backslash W$, contrary to the supposition that $W$ is open-and-closed.

We prove that $\cap_{\alpha<\Omega} V_{\alpha}$ is nonvoid. For $x$ in $G$ and $\alpha$ in $A$, we define $N(x, \alpha)$ to be the number of elements in the finite set $\{\beta \in A: \beta<\alpha$ and $\left.x_{\beta}=-1\right\}$. For $\alpha \in A$, we define

$$
n_{\alpha}=\inf _{x \in V_{\alpha}} N(x, \alpha) \text { and } J_{\alpha}=\left\{x \in V_{\alpha}: N(x, \alpha)=n_{\alpha}\right\} \text {. }
$$


Clearly $\varnothing \neq J_{\alpha} \subset V_{\alpha}$ for all $\alpha$. The integer-valued transfinite sequence $\left\{n_{\alpha}\right\}_{\alpha<\Omega}$ is nondecreasing because for $\alpha<\gamma$ we have

$$
n_{\alpha}=\inf _{x \in V_{\alpha}} N(x, \alpha) \leqq \inf _{x \in V_{\alpha}} N(x, \gamma) \leqq \inf _{x \in V_{\gamma}} N(x, \gamma)=n_{\gamma}
$$

It follows that the sequence $\left\{n_{\alpha}\right\}_{\alpha<\Omega}$ is eventually constant. There is, then, an integer $n_{0}$ and an $\alpha_{0}$ in $A$ for which $n_{\alpha}=n_{0}$ whenever $\alpha \geqq \alpha_{0}$. We next show that $\left\{J_{\alpha}\right\}_{\alpha \geqq \alpha_{\nu}}$ is a nonincreasing family of sets. Suppose that $\alpha_{0} \leqq \alpha<\gamma$ and $z \in J_{\gamma}$. Then $z \in J_{\gamma} \subset V_{\gamma} \subset V_{\alpha}$. Since also

$$
n_{\alpha} \leqq N(z, \alpha) \leqq N(z, \gamma)=n_{\gamma}=n_{0}=n_{\alpha},
$$

we see that $z \in J_{\alpha}$. Now let $Y$ consist of all elements $y$ of $G$ such that $y_{\beta}=1$ for all $\beta \geqq \alpha_{0}$. Then $Y$ is a countable set. Assume now that $\cap_{\alpha<\Omega} V_{\alpha}=\varnothing$, so that $\cap_{\alpha \geq \alpha_{0}} J_{\alpha}=\varnothing$. Then for each $y$ in $Y$ there is an $\alpha_{y} \geqq \alpha_{0}$ such that $y \notin J_{\alpha_{y}}$. Selecting $\gamma_{0}$ in $A$ larger than each $\alpha_{y}$, we find that $Y \cap J_{\gamma_{0}}=\varnothing$. Now choose $z$ in $J_{\gamma_{0}}$. Then $z$ also belongs to $J_{\alpha_{0}}$, so that $N\left(z, \gamma_{0}\right)=N\left(z, \alpha_{0}\right)=n_{0}$. Hence $z_{\beta}=1$ for $\alpha_{0} \leqq \beta<\gamma_{0}$. Define $w$ so that $w_{\beta}=z_{\beta}$ for $\beta<\gamma_{0}$ and $w_{\beta}=1$ for $\beta \geqq \gamma_{0}$. Clearly $w$ belongs to $Y$. Since $z \in J_{\gamma_{0}} \subset V_{\gamma_{0}}$, we have $w \in z H_{\gamma_{0}} \subset V_{\gamma_{0}}$. Also $N\left(w, \gamma_{0}\right)=N\left(z, \gamma_{0}\right)=n_{0}$, so that $w \in J_{\gamma_{0}}$. That is, $w$ belongs to $Y \cap J_{\gamma_{0}}$, contrary to the relation $Y \cap J_{\gamma_{0}}=\varnothing$. Thus $\cap_{\alpha<\Omega} V_{\alpha} \neq \varnothing$, and we conclude that $G$ has property $U$.

REMARK. It may be interesting to note that the group discussed above is Lindelof (and hence normal). To see this, assume that $\mathscr{C}$ is a cover of $G$ by basic open sets, and that $\mathscr{C}$ admits no countable subcover. For $\alpha \in A$, let $\mathscr{U}_{\alpha}$ consist of all elements of $\mathscr{U}$ which are translates of some $H_{\beta}$ where $\beta \leqq \alpha$. Since each $\mathscr{L}_{\alpha}$ is countable, no $\mathscr{U}_{\alpha}$ is a cover for $G$. Let $U_{\alpha}=\cup \mathscr{C}_{\alpha}$. Then $\left\{U_{\alpha}\right\}_{\alpha<\Omega}$ is a nondecreasing sequence of proper subsets of $G$, and each $U_{\alpha}$ is a union of cosets of $H_{\alpha}$. Let $V_{\alpha}=G \backslash U_{\alpha}$. As in Example 3.2 above, we have $\cap_{\alpha<\Omega} V_{\alpha} \neq$ $\varnothing$ : hence $\mathscr{U}$ does not cover $G$.

One may wonder whether Example 3.2 is typical of topological groups that are $P$-spaces: Do all topological groups that are $P$-spaces satisfy property $U$ ? The next theorem and the examples following it make Example 3.2 appear atypical.

THEOREM 3.3. Let $G$ be a nondiscrete topological group. If $G$ admits a base $\mathscr{H}$ at the identity consisting of open subgroups such that card $(G / K) \geqq$ card $\mathscr{\mathscr { C }}$ for some $K$ in $\mathscr{K}$, then $G$ does not have property $U$.

Proof. We may clearly suppose that $H \subset K$ for all $H$ in $\mathscr{C}$. By the cardinality hypothesis, there exists a subset $\left\{x_{H}\right\}_{B \in \mathscr{C}}$ of $G$, indexed 
by $\mathscr{K}$, such that $\left\{x_{H} K\right\}_{H \in \mathscr{C}}$ is a family of distinct cosets of $K$. Let $W=U_{H \in \mathscr{H}} x_{H} H$. Clearly $W$ is open, and $W$ is closed because

$$
G \backslash W=\left(G \backslash \bigcup_{H \in \mathscr{H}} x_{H} K\right) \cup \bigcup_{H \in \mathscr{H}}\left(x_{H} K \backslash x_{H} H\right) .
$$

Therefore $\psi_{W}$ is continuous; we next show that $\psi_{W}$ is not left uniformly continuous. Indeed, suppose that there exists an $H_{0} \in \mathscr{Y}$ such that $x^{-1} y \in H_{0}$ implies $x \in W$ if and only if $y \in W$. Since $G$ is nondiscrete, there exists an $H$ in $\mathscr{C}$ such that $H \subset H_{0}$ and $H \neq H_{0}$. If $y$ is chosen so that $y \in x_{H} H_{0} \mid x_{H} H$, then $y \in x_{H} K \backslash x_{H} H \subset G \backslash W$. Since $x_{H}^{-1} y \in H_{0}$, we also have $x_{H} \in G \backslash W$. This contradicts the fact that $x_{H} \in x_{H} H \subset W$.

EXAMPLES 3.4. Let $\ell$ be a cardinal number less than the first strongly inaccessible cardinal ${ }^{1}$. Let $G$ be the algebraic group $\{1,-1\}^{\mu}=$ $\prod_{\alpha \in A}\{1,-1\}_{\alpha}$, where the index set $A$ is ordered according to the least ordinal having cardinality $\mu$. Let the subgroups

$$
H_{r}=\left\{x \in G: x_{\beta}=1 \text { for all } \beta<\alpha\right\}
$$

and all their translates be a basis for a topology on $G$.

If $\nu$ denotes the smallest cardinal number which is the cardinal number of some cofinal subset of $A$, then evidently $\nu$ is the minimal cardinality of a base at the identity of $G$. If $\mu$ is chosen so that $\nu>\boldsymbol{S}_{0}$, then the nondiscrete topology imposed upon $G$ is clearly a $P$-space topology, and under the condition $\nu>\boldsymbol{\aleph}_{0}$ we can show that $G$ does not have property $U$.

To do this, suppose first that $2^{\kappa}<\nu$ whenever $\kappa<\nu$. Then (from 12.4-12.6 of [5]) there is a set $\left\{\nu_{\lambda}\right\}_{\lambda \in 1}$ of cardinal numbers such that card $A<\nu, \nu_{\lambda}<\nu$ for each $\lambda$ in $A$, and $\sup \nu_{\lambda}=\nu$. Since there is then a cofinal set $\left\{\alpha_{\lambda}: \lambda \in A\right\}$ in $A$ indexed by $\Lambda$, contrary to the minimality of $\nu$, we conclude that $2^{\kappa} \geqq \nu$ for some $\kappa<\nu$. Now let $\mathscr{C}$ be a basis of open subgroups at the identity for which card $\mathscr{H}=\nu$, and choose $\beta \in A$ so that $H_{\beta} \in \mathscr{H}$ and $\operatorname{card}\{\alpha \in A: \alpha<\beta\} \geqq \kappa$. Then

$$
\operatorname{card}(G / H) \geqq 2^{\kappa} \geqq \nu,
$$

so that $G$ does not have property $U$ by 3.3 .

4. Related concepts. Much of our earlier work is summarized in the following theorem. The symbol $\beta G$ denotes the Stone-Čech compactification of the (completely regular) space $G$; it is, to within a homeomorphism leaving $G$ fixed pointwise, the only compactification

1 A cardinal number is said to be strongly inaccessible if it is an uncountable cardinal whose set of predecessors is closed under the standard operations of cardinal arithmetic. It is not known whether any strongly inaccessible cardinal number exists. 
of $G$ to which each bounded continuous real-valued function on $G$ admits a continuous extension. The amusing suggestion that $G$ might induce a topological group structure on $\beta G$ is not original with us: The appearance of this phenomenon was explicitly pointed out in [6] by Glicksberg in connection with the Corson $\Sigma$-space mentioned earlier.

The implication $(\mathrm{b}) \Rightarrow(\mathrm{g})$ of 4.1 below was given in [6], and Glicksberg asked whether or not the implication $(\mathrm{a}) \Rightarrow(\mathrm{g})$ is valid. Our proof of its validity does not depend upon the results of [6].

If the identity in a topological group $G$ admits a neighborhood $U$ which is bounded (in the sense that for each nonempty open subset $V$ of $G$ there is a finite set $F$ such that $U \subset F V$ ), then $G$ is said to be locally bounded.

We remark finally that additional conditions equivalent to those listed below may be obtained by replacing the expression " $G$ has property $U$ " when it appears by the expression " $G$ has property $B U$."

THEOREM 4.1. For a topological group G, conditions (a) through (g) are equivalent, and each implies (h). If in addition $G$ is nondiscrete, then all eight conditions are equivalent.

(a) $G$ is pseudocompact;

(b) $G \times G$ is pseudocompact;

(c) $G$ is pseudocompact and has property $U$;

(d) $G$ is totally bounded and has property $U$;

(e) $G$ is totally bounded and $\beta G=\bar{G}$;

(f) $\beta G$ admits a topological group structure relative to which the inclusion mapping of $G$ into $\beta G$ is a topological isomorphism;

(g) every continuous real-valued function on $G$ is almost periodic;

(h) $G$ is locally bounded and has property $U$.

Proof. Theorem 1.4 gives the implication $(a) \Rightarrow(b)$, and the converse follows from the fact that the continuous image of a pseudocompact space is pseudocompact. The implications $(a) \Rightarrow(c),(c) \Rightarrow(d)$, and $(d) \Rightarrow$ (a) are $1.5,1.1$, and 2.7 respectively, while the implication (a) $\Rightarrow$ (e) follows from 1.1 and the implication $(a) \Rightarrow(e)$ of 1.2. That $(e) \Rightarrow(f)$ is obvious, and the implication $(f) \Rightarrow$ (d) follows from 2.8 .

We have shown so far that the first six conditions listed are equivalent.

To deduce (g), suppose that (a) and (e) hold and let $f$ be any continuous real-valued function on $G$. Being bounded, $f$ admits a continuous real-valued extension to $\beta G$. A routine computation, based on the fact that every continuous real-valued function on the compact group $\beta G$ is almost periodic on $\beta G$, shows that $f$ is almost periodic on $G$.

To see that (g) implies (a), let $f$ be any continuous real-valued 
function on $G$ and let $F$ be a finite subset of $G$ with the property that for each $x$ in $G$ there exists $y$ in $F$ such that $|f(x z)-f(y z)|<1$ whenever $z \in G$. Then for each $x$ in $G$ we have

$$
|f(x)|=|f(x e)|<\max _{y \in F}|f(y)|+1 \text {. }
$$

Since the implication $(\mathrm{d}) \Rightarrow(\mathrm{h})$ is obvious, we may complete the proof by supposing that $G$ is nondiscrete and deducing (d) from (h). If (h) holds but (d) fails, then $G$ is a $P$-space by 2.2. Let $U$ be a bounded neighborhood of $e$ and let $\left\{x_{k}\right\}$ be an infinite set of distinct points in $U$. For each pair $(m, n)$ of distinct positive integers there is a neighborhood $V_{m, n}$ of the identity such that $x_{m} \notin x_{n} V_{m, n}$. Choosing a symmetric neighborhood $V$ of the identity such that

$$
V^{2} \subset \bigcap_{(m, n)} V_{m, n}
$$

we see easily that no set of the form $x V$ can contain more than one of the points $x_{k}$. Thus there exists no finite subset $F$ of $G$ for which $U \subset F V$.

In the discussion and example which follow we will say that a uniform space on which each real-valued uniformly continuous function is bounded has property $U B$. Clearly any totally bounded uniform space has property $U B$, and Atsuji gives in [1] an example of a connected metric space that is not totally bounded but which has property $U B$. Further metric examples are given in exercises 15.D and 15.L of [5]. Although Atsuji in Theorem 7 of [2] characterizes uniform spaces with property $U B$ by means of a chainability condition, the following question has not so far as we can determine been treated in the literature: Must a topological group with property $U B$ be totally bounded? We now answer this question in the negative.

EXAMPLE 4.2. Let $T$ denote the circle group and let $G$ be the algebraic group $T^{\aleph_{0}}=\prod_{k=1}^{\infty} T_{k}$. Defining

$$
d(x, y)=\sup _{k}\left|x_{k}-y_{k}\right|
$$

for each pair of points $x, y$ in $G$, we obtain a metric topology on $G$ under which $G$ is a topological group. To see that $G$ has property $U B$, let $f$ be a uniformly continuous real-valued function on $G$ and find $\delta>0$ such that $|f(x)-f(y)|<1$ whenever $d(x, y)<\delta$. Choose an integer $m$ so that, given any point $t$ in $T$, there is a sequence $1=$ $t^{0}, t^{1}, \cdots, t^{m}=t$ in $T$ such that $\left|t^{j+1}-t^{j}\right|<\delta / 2$ for $0 \leqq j \leqq m-1$. We will show that $|f(x)| \leqq|f(e)|+m$ for all $x$ in $G$. For a fixed $x$ in $G$, select for each integer $k>0$ a sequence $1=x_{k}^{0}, x_{k}^{1}, \cdots, x_{k}^{m}=x_{k}$ in $T$ such that $\left|x_{k}^{j+1}-x_{k}^{j}\right|<\delta / 2$. The finite sequence $x^{0}, x^{1}, \cdots, x^{m}$ in $G$ has the property that 


$$
d\left(x^{j+1}, x^{j}\right) \leqq \delta / 2<\delta \quad \text { for } \quad 0 \leqq j \leqq m-1 .
$$

Hence $\left|f\left(x^{j+1}\right)-f\left(x^{j}\right)\right|<1$ for $0 \leqq j \leqq m-1$, so that $|f(x)-f(e)| \leqq$ $m$. Thus $G$ has property $U B$.

To see that $G$ is not totally bounded, let $W$ be the open set $\{x \in G: d(x, e)<1 / 2\}$. Regarding $G$ as the usual compact topological group $T^{\aleph_{0}}$ with its Haar measure, we see that the $G_{\delta}$ set $W$ has Haar measure 0 . It follows that no finite number of translates of $W$ can cover $G$.

\section{REFERENCES}

1. Masahiko Atsuji, Uniform continuity of continuous functions of metric spaces, Pacific J. Math. 8 (1958), 11-16.

2. Masahiko Atsuji, Uniform continuity of continuous functions on uniform spaces, Canad. J. Math. 13 (1961), 657-663.

3. H. H. Corson, Normality in subsets of product spaces, Amer. J. Math. 81 (1959), 785-796.

4. Zdenèk Frolík, The topological product of two pseudocompact spaces, Czechoslovak Math. J. 10 (1960), 339-349.

5. Leonard Gillman and Meyer Jerison, Rings of continuous functions, D. Van Nostrand Company, Princeton, 1960.

6. Irving Glicksberg, Stone-Čech compactifications of products, Trans. Amer. Math. Soc. 90 (1959), 369-382.

7. Edwin Hewitt and Kenneth A. Ross, Abstract harmonic analysis I, Springer-Verlag, Heidelberg, 1963.

8. J. M. Kister, Uniform continuity and compactness in topological groups, Proc. Amer. Math. Soc. 13 (1962), 37-40.

9. Kenneth A. Ross and Karl Stromberg, Baire sets and Baire measures, Archiv för Math. 8 (1965), 151-160.

10. André Weil, Sur les espaces à structure uniforme et sur la topologie générale, Publ. Math. Univ. Strasbourg, Hermann, Paris, 1937.

UNIVERSITY OF ROCHESTER

UNIVERSITY OF MASSACHUSETTS

UNIVERSITY OF OREGON 


\section{PACIFIC JOURNAL OF MATHEMATICS}

\section{EDITORS}

\author{
H. SAMmLSON \\ Stanford University \\ Stanford, California \\ R. M. BLUMENTHAL \\ University of Washington \\ Seattle, Washington 98105
}

\author{
*J. DUGUNDJI \\ University of Southern California \\ Los Angeles, California 90007
}

RICHARD ARENS

University of California

Los Angeles, California 90024

\section{E. F. BECKENBACH \\ B. H. NEUMANN \\ ASSOCIATE EDITORS}

\section{SUPPORTING INSTITUTIONS}

\author{
UNIVERSITY OF BRITISH COLUMBIA \\ CALIFORNIA INSTITUTE OF TECHNOLOGY \\ UNIVERSITY OF CALIFORNIA \\ MONTANA STATE UNIVERSITY \\ UNIVERSITY OF NEVADA \\ NEW MEXICO STATE UNIVERSITY \\ OREGON STATE UNIVERSITY \\ UNIVERSITY OF OREGON \\ OSAKA UNIVERSITY \\ UNIVERSITY OF SOUTHERN CALIFORNIA
}

\author{
STANFORD UNIVERSITY \\ UNIVERSITY OF TOKYO \\ UNIVERSITY OF UTAH \\ WASHINGTON STATE UNIVERSITY \\ UNIVERSITY OF WASHINGTON \\ * * * \\ AMERICAN MATHEMATICAL SOCIETY \\ CHEVRON RESEARCH CORPORATION \\ TRW SYSTEMS \\ NAVAL ORDNANCE TEST STATION
}

\footnotetext{
Mathematical papers intended for publication in the Pacific Journal of Mathematics should be typewritten (double spaced). The first paragraph or two must be capable of being used separately as a synopsis of the entire paper. It should not contain references to the bibliography. Manu. scripts may be sent to any one of the four editors. All other communications to the editors should be addressed to the managing editor, Richard Arens at the University of California, Los Angeles, California 90024.

50 reprints per author of each article are furnished free of charge; additional copies may be obtained at cost in multiples of 50 .
}

The Pacific Journal of Mathematics is published monthly. Effective with Volume 16 the price per volume (3 numbers) is $\$ 8.00$; single issues, $\$ 3.00$. Special price for current issues to individual faculty members of supporting institutions and to individual members of the American Mathematical Society: $\$ 4.00$ per volume; single issues $\$ 1.50$. Back numbers are available.

Subscriptions, orders for back numbers, and changes of address should be sent to Pacific Journal of Mathematics, 103 Highland Boulevard, Berkeley 8, California.

Printed at Kokusai Bunken Insatsusha (International Academic Printing Co., Ltd.), No. 6, 2-chome, Fujimi-cho, Chiyoda-ku, Tokyo, Japan.

PUBLISHED BY PACIFIC JOURNAL OF MATHEMATICS, A NON-PROFIT CORPORATION

The Supporting Institutions listed above contribute to the cost of publication of this Journal, but they are not owners or publishers and have no responsibility for its content or policies.

* Paul A. White, Acting Editor until J. Dugundji returns. 


\section{Pacific Journal of Mathematics}

\section{Vol. 16, No. $3 \quad$ BadMonth, 1966}

Gert Einar Torsten Almkvist, Stability of linear differential equations with

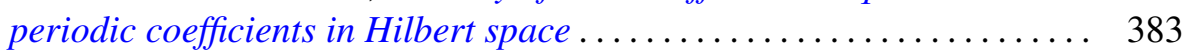

Richard Allen Askey and Stephen Wainger, A transplantation theorem for ultraspherical coefficients ................................ 393

Joseph Barback, Two notes on regressive isols .................. 407

Allen Richard Bernstein and Abraham Robinson, Solution of an invariant subspace problem of K. T. Smith and P. R. Halmos .............. 421

P. R. Halmos, Invariant subspaces of polynomially compact operators . . . . 433

Leon Bernstein, New infinite classes of periodic Jacobi-Perron algorithms.................................... 439

Richard Anthony Brualdi, Permanent of the direct product of matrices .... . 471

W. Wistar (William) Comfort and Kenneth Allen Ross, Pseudocompactness and uniform continuity in topological groups .................. 483

James Michael Gardner Fell, Algebras and fiber bundles . . . . . . . . . . . . 497

Alessandro Figà-Talamanca and Daniel Rider, A theorem of Littlewood and lacunary series for compact groups ..................... 505

David London, Two inequalities in nonnegative symmetric matrices...... 515

Norman Jay Pullman, Infinite products of substochastic matrices ........ 537

James McLean Sloss, Reflection and approximation by interpolation along

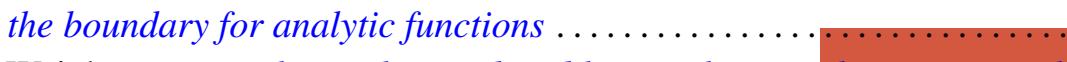

Carl Weinbaum, Visualizing the word problem, with an application to sixth groups................................... 